\title{
Prenatal exposure to antibiotics and wheezing in infancy: a birth cohort study
}

\author{
Maja Popovic ${ }^{1,2}$, Franca Rusconi $^{3}$, Daniela Zugna ${ }^{1,2}$, Claudia Galassi $^{2,4}$, \\ Franco Merletti ${ }^{1,2,4}$, Enrica Migliore ${ }^{1,2,4}$, Morena Trevisan ${ }^{2,4}$, Tiziana Nannelli ${ }^{5}$, \\ Luigi Gagliardi ${ }^{6}$ and Lorenzo Richiardi ${ }^{1,2,4}$
}

\author{
Affiliations: \\ ${ }^{1}$ Dept of Medical Sciences, University of Turin, Turin, Italy. \\ ${ }^{2} \mathrm{CPO}$ Piemonte, Turin, Italy. \\ 3Unit of Epidemiology, "Anna Meyer" Children's University Hospital, Florence, Italy. \\ ${ }^{4}$ AOU Città della Salute e della Scienza, Turin, Italy. \\ ${ }^{5}$ Dept of Clinical and Experimental Medicine, University of Florence, Florence, Italy. \\ ${ }^{6}$ Dept of Woman and Child Health, Paediatrics and Neonatology Division, Ospedale Versilia, Viareggio, Italy.
}

Correspondence: Maja Popovic, Dept of Medical Sciences, University of Turin, Via Santena 7, 10126 Turin, Italy. E-mail: maja_popovicahotmail.com

ABSTRACT The role of prenatal antibiotic exposure in the development of childhood wheezing is debated. We evaluated whether this association could potentially be explained by confounding factors.

Antibiotic use in the first and third trimester of pregnancy, wheezing in children aged $\leqslant 18$ months and confounding factors were assessed in singletons participating in the NINFEA (Nascita e Infanzia: gli Effetti dell'Ambiente) birth cohort ( $\mathrm{n}=3530$ for first-trimester exposure and $\mathrm{n}=3985$ for third-trimester exposure).

There was no evidence of an association between antibiotic exposure in the first trimester of pregnancy and ever-wheezing (adjusted risk ratio (RR) 1.02, 95\% CI 0.80-1.30) or recurrent wheezing (RR 0.99, 95\% CI 0.54-1.82). For the third-trimester exposure, the crude RRs (95\% CI) of ever-wheezing and recurrent wheezing were $1.34(1.10-1.64)$ and $2.72(1.80-4.11)$, respectively, which decreased to $1.12(0.90-1.39)$ and 2.09 (1.32-3.29) after adjustment. The RRs of wheezing after genitourinary infections during pregnancy were increased independently of antibiotic treatment.

In conclusion, the association between prenatal antibiotic exposure and infant wheezing could be largely explained by confounding factors, in particular respiratory infections during pregnancy. An excess risk of wheezing after antibiotic exposure during the third trimester of pregnancy remains after adjustment.

@ERSpublications

Confounders explain increased risk of infant wheeze after antibiotic exposure in the first but not third trimester http://ow.ly/Tu1U0

This article has supplementary material available from erj.ersjournals.com

A press release for this article is available from erj.ersjournals.com/press

Received: Oct 152014 | Accepted after revision: Oct 022015 | First published online: Dec 032015

Support statement: This study was funded by the Compagnia di San Paolo Foundation. Funding information for this article has been deposited with FundRef.

Conflict of interest: None declared.

Copyright OERS 2016 


\section{Introduction}

There is growing evidence of the key role of the prenatal environment in the development of childhood wheezing [1,2]. One of the well-known risk factors implicated in the development of wheezing and an associated diminished lung function is maternal smoking during pregnancy [2,3]. The role of other prenatal exposures, including pregnancy complications or air pollution exposure, is less clear [4].

In recent years, several studies reported an increased risk of wheezing and asthma in infants and children after prenatal antibiotic exposure [5-18]. An imbalance of maternal vaginal and bowel bacterial flora caused by antibiotic treatment could lead to an abnormal early colonisation of infant gut and airway through vertical transmission during birth. It has been hypothesised that an altered composition of the infant's microbiota might cause a disruption of mucosal immune tolerance and lead to the development of T-helper type 2 mediated diseases $[5,19,20]$.

Alternatively, the association between antibiotic use during pregnancy and childhood wheezing/asthma might stem from various confounding factors [21]. Potential confounders include risk factors for both maternal and childhood infections and wheezing such as maternal smoking and asthma, both associated with a greater risk of respiratory infections, and perinatal infections, e.g. chorionamnionitis, which might be treated with antibiotics [22].

Within the framework of a mother-child cohort study, we evaluated the association between antibiotic use during pregnancy and the risk of wheezing up to the age of 18 months in children. We aimed in particular at investigating to what extent these associations might be explained by confounding factors.

\section{Methods}

\section{Study population}

NINFEA (Nascita e Infanzia: gli Effetti dell'Ambiente; Birth and Childhood: Effects of the Environment) is an ongoing Italian internet-based birth cohort established in 2005 (www.progettoninfea.it) [23]. Members of the cohort are children of mothers who have access to the internet and enough knowledge of Italian to complete online questionnaires. Recruitment is conducted actively through obstetrics clinics, and passively via the internet and the media [24]. 75\% of participants are recruited actively, $20 \%$ passively and 5\% comprise both modes. Participants complete the baseline questionnaire at any time during pregnancy and another four questionnaires at 6 and 18 months after delivery and when children turn 4 and 7 years of age.

We used the 2015.03 version of the NINFEA database and the information from the first three questionnaires. The study population consisted of 4421 singletons with information on occurrence of wheezing. Analyses regarding the first trimester exposure were restricted to 3812 children as we excluded 609 children born to mothers who completed the first questionnaire before the end of the first trimester of pregnancy. The NINFEA response rate at the 18 -month questionnaire is $84 \%$.

The study was approved by the ethical committee of the San Giovanni Battista Hospital and CTO/CRF/ Maria Adelaide Hospital (Turin, Italy) (approval N.0048362 and following amendments).

\section{Exposure variables}

Antibiotic exposure in the first trimester was assessed using the questionnaire completed after the end of the first trimester of pregnancy, while third-trimester exposure was assessed using the questionnaire completed 6 months after delivery. Second-trimester exposure was not evaluated in this study, as such analysis would have required an exclusion of all participants who completed the first questionnaire before the end of the second trimester, leading to a great reduction of the sample size. Duration of antibiotic use was also analysed. Questionnaires included additional information on any medication used during pregnancy to treat a number of chronic conditions, listed in a pre-specified checklist, from which we selected those that could represent an indication for antibiotic use (namely, genitourinary infections, including recurrent urinary infections, Chlamydia infections and kidney stones). Three categories were identified: 1) females with at least one of the three diseases not treated with antibiotics; 2) females with at least one of the diseases treated with antibiotics; and 3) females without any of the three diseases.

The exact wording of the questions used in the study is reported in online supplementary table S1.

\section{Outcome variables}

Ever-wheezing was defined as at least one episode of wheezing or whistling in the chest in the first 6 months or between 6 and 18 months of life, while recurrent wheezing was defined as wheezing or whistling in the chest reported both in the first 6 months and between 6 and 18 months of life. The exact wording of the questions used in the study is reported in online supplementary table S2. 
TABLE 1 Main characteristics of the study population

\begin{tabular}{|c|c|c|c|c|}
\hline & \multicolumn{2}{|c|}{$\begin{array}{l}\text { First trimester } \\
\text { antibiotics }\end{array}$} & \multicolumn{2}{|c|}{$\begin{array}{l}\text { Third trimester } \\
\text { antibiotics }\end{array}$} \\
\hline & No & Yes & No & Yes \\
\hline Subjects & 3301 (93.5) & $229(6.5)$ & $3729(93.6)$ & $256(6.4)$ \\
\hline \multicolumn{5}{|l|}{ Maternal educational level } \\
\hline University degree or higher & $2031(61.5)$ & $150(65.5)$ & $2292(61.5)$ & 165 (64.5) \\
\hline Secondary school & $1132(34.3)$ & $69(30.1)$ & $1271(34.1)$ & $82(32.0)$ \\
\hline Primary school or less & $138(4.2)$ & $10(4.4)$ & $166(4.4)$ & $9(3.5)$ \\
\hline \multicolumn{5}{|l|}{ Maternal age at childbirth years } \\
\hline$<30$ & $654(19.8)$ & $44(19.2)$ & $727(19.5)$ & $43(16.8)$ \\
\hline $30-34$ & $1462(44.3)$ & $86(37.5)$ & $1634(43.8)$ & $110(43.0)$ \\
\hline $35-39$ & $977(29.6)$ & $70(30.6)$ & $1113(29.9)$ & $86(33.6)$ \\
\hline$\geqslant 40$ & $208(6.3)$ & 29 (12.7) & $255(6.8)$ & $17(6.6)$ \\
\hline \multicolumn{5}{|c|}{ Maternal asthma/asthmatic bronchitis } \\
\hline Yes & $247(7.5)$ & $20(8.7)$ & $276(7.4)$ & $25(9.8)$ \\
\hline No & 3054 (92.5) & 209 (91.3) & $3453(92.6)$ & $231(90.2)$ \\
\hline \multicolumn{5}{|l|}{ Siblings } \\
\hline Yes & 975 (29.5) & $95(41.5)$ & $1200(32.2)$ & 102 (39.8) \\
\hline No & $2326(70.5)$ & $134(58.5)$ & $2529(67.8)$ & $154(60.2)$ \\
\hline \multicolumn{5}{|l|}{ Maternal smoking in pregnancy } \\
\hline Yes & $257(7.8)$ & $20(8.7)$ & $289(7.7)$ & $20(7.8)$ \\
\hline No & $3044(92.2)$ & $209(91.3)$ & 3440 (92.3) & $236(92.2)$ \\
\hline \multicolumn{5}{|c|}{ Maternal paracetamol use in pregnancy } \\
\hline Yes & $1752(53.1)$ & $148(64.6)$ & 1989 (53.3) & $174(68.0)$ \\
\hline No & $1549(46.9)$ & $81(35.4)$ & $1740(46.7)$ & $82(32.0)$ \\
\hline \multicolumn{5}{|c|}{ Maternal respiratory infections in pregnancy } \\
\hline \multicolumn{5}{|c|}{ Otitis and/or sinusitis and/or throat infections } \\
\hline Yes & $278(8.4)$ & $60(26.2)$ & $201(5.4)$ & $66(25.8)$ \\
\hline No & $3023(91.6)$ & $169(73.8)$ & $3528(94.6)$ & $190(74.2)$ \\
\hline \multicolumn{5}{|l|}{ Bronchitis and/or flu } \\
\hline Yes & $175(5.3)$ & $33(14.4)$ & $153(4.1)$ & 48 (18.7) \\
\hline No & $3126(94.7)$ & $196(85.6)$ & $3576(95.9)$ & $208(81.3)$ \\
\hline \multicolumn{5}{|l|}{ Common cold } \\
\hline Yes & $621(18.8)$ & $48(21.0)$ & $463(12.4)$ & $67(26.2)$ \\
\hline No & $3680(81.2)$ & $181(79.0)$ & $3266(87.6)$ & $189(73.8)$ \\
\hline \multicolumn{5}{|c|}{ Other maternal infections in pregnancy } \\
\hline \multicolumn{5}{|c|}{ Urinary infections and/or urinary burning } \\
\hline Yes & $122(3.7)$ & $46(20.1)$ & $202(5.4)$ & 70 (27.3) \\
\hline No & $3179(96.3)$ & $183(79.9)$ & $3527(94.6)$ & $186(72.7)$ \\
\hline \multicolumn{5}{|l|}{ Vaginal infections } \\
\hline Yes & $194(5.9)$ & $62(27.1)$ & 353 (9.5) & $80(31.2)$ \\
\hline No & $3107(94.1)$ & $167(72.9)$ & 3373 (90.5) & $176(68.8)$ \\
\hline \multicolumn{5}{|l|}{ Fever $>38^{\circ} \mathrm{C}$} \\
\hline Yes & $90(2.7)$ & $22(9.6)$ & $58(1.6)$ & $29(11.3)$ \\
\hline No & $3211(97.3)$ & $207(90.4)$ & $3671(98.4)$ & $227(88.7)$ \\
\hline \multicolumn{5}{|l|}{ Mode of delivery } \\
\hline Vaginal & 2254 (74.3) & $136(65.4)$ & $2548(74.0)$ & 159 (67.7) \\
\hline Caesarean section & $779(25.7)$ & $72(34.6)$ & $895(26.0)$ & 76 (32.3) \\
\hline \multicolumn{5}{|l|}{ Wheezing up to 6 months } \\
\hline Yes & $220(7.1)$ & $22(10.6)$ & $268(7.3)$ & $34(13.4)$ \\
\hline No & $2887(92.9)$ & $185(89.4)$ & 3410 (92.7) & $219(86.6)$ \\
\hline \multicolumn{5}{|l|}{ Ever-wheezing up to 18 months } \\
\hline Yes & $690(20.9)$ & $60(26.2)$ & $803(21.5)$ & 74 (28.9) \\
\hline No & $2611(79.1)$ & $169(73.8)$ & $2926(78.5)$ & $182(71.1)$ \\
\hline Recurrent wheezing & & & & \\
\hline Yes & $118(3.8)$ & $12(5.7)$ & $134(3.8)$ & $24(10.4)$ \\
\hline No & $2989(96.2)$ & 200 (94.3) & $3371(96.2)$ & $207(89.6)$ \\
\hline
\end{tabular}

Data are presented as $\mathrm{n}(\%)$. 
In the 18-month questionnaire mothers were additionally asked to report if a paediatrician had ascertained wheezing episodes occurring between 6 and 18 months of age; doctor confirmation was reported in $89.8 \%$ of cases.

\section{Confounding factors}

Three sets of potential confounders were pre-selected: 1) core confounders, including maternal age and educational level at delivery, maternal smoking and paracetamol use during pregnancy, number of siblings of the child and maternal history of asthma; 2) respiratory infections during pregnancy including otitis, sinusitis, throat infections, bronchitis, flu and common cold; and 3) other infections during pregnancy that may represent an indication for antibiotic use, including vaginal and urinary infections as well as episodes of fever $>38^{\circ} \mathrm{C}$ as a nonspecific indicator for infectious diseases. The confounding factors were coded as shown in table 1.

\section{Statistical analyses}

We used a complete case analysis approach which implied an exclusion of $<10 \%$ of the subjects. The analyses included 3530 and 3319 subjects for the first-trimester exposure, and 3985 and 3736 subjects for the third-trimester exposure for ever-wheezing and recurrent wheezing, respectively. Crude and adjusted risk ratios (RR) with 95\% confidence intervals were estimated using a Poisson regression model [25]. Since mothers could participate in the NINFEA cohort with more than one pregnancy we estimated robust variance using clustered sandwich estimators to allow for intragroup correlation. The amount of confounding by each confounders' set, as well as by the single factors, was quantified by the change-in-estimate criterion [26]. As we had no information on the antibiotic spectrum used, we performed an additional analysis by excluding mothers who took antibiotics to treat genitourinary infections ( $\mathrm{n}=21$ for the first trimester and $\mathrm{n}=51$ for the third trimester) and compared the remaining mothers with those unexposed. We further restricted the analyses to mothers who used antibiotics for $\geqslant 3$ days with the purpose of reducing potential exposure misclassification. The presence of collinearity between antibiotic exposure and infections during pregnancy was checked by looking at the variance inflation factors for the final models [27].

Females undergoing Caesarean sections are typically pretreated with antibiotics [28], but are either unaware of it or simply underreport this exposure. As Caesarean sections might be associated with wheezing/asthma in childhood [29], in order to reduce possible misclassification we conducted a sensitivity analysis restricted to the vaginally delivered children ( $\mathrm{n}=2707$ for ever-wheezing and $\mathrm{n}=2536$ for recurrent wheezing).

Finally, in order to evaluate confounding by indication we compared children of mothers with genitourinary infections who were treated with antibiotics with untreated ones. For completeness, we also estimated the association between genitourinary infections (regardless of antibiotic treatment) and infant wheezing.

All analyses were performed using Stata 13 (Stata Corporation, College Station, TX, USA).

\section{Results}

Main characteristics of the study population are presented in table 1 . The prevalence of antibiotic use was $6.5 \%$ and $6.4 \%$ in the first and third trimesters, respectively, with an overlap of $18.5 \%$ between these two

TABLE 2 Associations between prenatal exposure to antibiotics (users versus nonusers) and wheezing outcomes in children aged $\leqslant 18$ months

\begin{tabular}{|c|c|c|c|c|c|c|}
\hline Outcomes & Cases & Exposed cases & $\mathbf{R R}_{\text {crude }}$ & $\mathbf{R}_{\mathbf{a d j}_{1}} \#$ & $\mathbf{R R}_{\mathrm{adj} 2}$ ๆ & $\mathbf{R R}_{\mathrm{adj} 3^{+}}{ }^{+}$ \\
\hline \multicolumn{7}{|l|}{ First trimester } \\
\hline Ever-wheezing & 750 & $60(8.0)$ & $1.25(1.00-1.57)$ & $1.14(0.91-1.43)$ & $1.07(0.85-1.35)$ & $1.02(0.80-1.30)$ \\
\hline \multicolumn{7}{|l|}{ Third trimester } \\
\hline Ever-wheezing & 877 & $74(8.4)$ & $1.34(1.10-1.64)$ & $1.25(1.02-1.52)$ & $1.18(0.95-1.46)$ & $1.12(0.90-1.39)$ \\
\hline Recurrent wheezing & 158 & $24(15.2)$ & $2.72(1.80-4.11)$ & $2.36(1.57-3.54)$ & $2.25(1.44-3.53)$ & $2.09(1.32-3.29)$ \\
\hline
\end{tabular}

Data are presented as $\mathrm{n}, \mathrm{n}(\%)$ or risk ratio (RR) $(95 \% \mathrm{Cl}) . \mathrm{RR}_{\mathrm{adj}}$ : adjusted risk ratio. \#: adjusted for maternal educational level, maternal age at birth, smoking in pregnancy, siblings, paracetamol use in pregnancy and maternal asthma or asthmatic bronchitis; ${ }^{\text {ๆ: }}$ adjusted as RR $\mathrm{Rdj}$ and for maternal respiratory infections (bronchitis, flu, throat infections, otitis, sinusitis and common cold); ${ }^{+}$: adjusted as $\mathrm{RR}_{\mathrm{adj} 2}$ and for other maternal infections (urinary tract infections and vaginal infections) and fever $>38^{\circ} \mathrm{C}$. 
exposures. Paracetamol use during pregnancy, Caesarean delivery and presence of siblings of the index child were more frequent among the exposed subjects (both trimesters $\mathrm{p}<0.03$ ), as well as infections and fever $>38^{\circ} \mathrm{C}$ (both trimesters $\mathrm{p}<0.001$ ). Mothers exposed in the first trimester were older compared with the unexposed subjects $(\mathrm{p}=0.002)$. Children exposed in the third trimester suffered more often from both ever-wheezing and recurrent wheezing than those unexposed ( $p=0.028$ and $p<0.001$, respectively).

The associations between prenatal antibiotic exposure and infant wheezing are reported in table 2. All crude estimates gradually attenuated after adjustment for each set of confounding factors. Only the association between recurrent wheezing and antibiotic exposure during the third trimester of pregnancy remained after adjustment ( $\mathrm{RR} 2.09 ; 95 \% \mathrm{CI}$ 1.32-3.29). In the third trimester, maternal respiratory infections were the strongest confounder of the association between prenatal antibiotic exposure and infant wheezing, and together with the core confounders had a strong confounding role in the first trimester (table 3). More precisely, maternal otitis, sinusitis and throat infections among respiratory infections, urinary infections among the other infections during pregnancy and presence of siblings (especially for the first-trimester exposure) among the core confounders had the strongest confounding effects on the associational estimates (online supplementary table S3). In addition, for all the associations between antibiotic use and infant wheezing the main confounding factors were positive, with the exception of fever which was a negative confounder for the associations involving the third-trimester exposure. All variance inflation factor values were below five, indicating a stable model with no evidence of multicollinearity problems.

Restricting the analyses to mothers treated with antibiotics other than those for genitourinary infections did not alter the results (data not shown).

In the sensitivity analysis intended to reduce potential exposure misclassification, restriction to mothers who used antibiotics during the first trimester for $\geqslant 3$ days yielded fully adjusted RRs (95\% CI) of 1.01 (0.78-1.30) for ever-wheezing and $1.03(0.54-1.95)$ for recurrent wheezing. In the same analysis for the third trimester exposure, fully adjusted RRs (95\% CI) of ever-wheezing and recurrent wheezing were 1.06 (0.84-1.34) and $1.88(1.16-3.05)$, respectively.

The analyses on the third-trimester antibiotics exposure restricted to children delivered vaginally yielded fully adjusted RRs (95\% CI) of $1.09(0.81-1.47)$ for ever-wheezing and $1.72(0.88-3.34)$ for recurrent wheezing.

We found no evidence of an increased risk of wheezing among the children of mothers who used antibiotics to treat genitourinary infections compared with the children of mothers who did not use antibiotics to treat them (table 4). However, the confidence intervals are wide due to the substantially smaller sample size of this subgroup of the cohort. When all the mothers with genitourinary infections during pregnancy were compared with those without infections, we found, for the first trimester, adjusted RRs (95\% CI) of 1.33 (0.97-1.81) for ever-wheezing and 2.43 (1.29-4.59) for recurrent wheezing, and for the third trimester, adjusted RRs (95\% CI) of 1.34 (1.10-1.62) for ever-wheezing and 2.16 (1.39-3.34) for recurrent wheezing (online supplementary table S4).

\section{Discussion}

Our results show positive crude associations between prenatal antibiotic exposure and infant wheezing, but the estimates decrease and often annul after adjustment, particularly for maternal infections during pregnancy. An increased risk of recurrent wheezing after antibiotic exposure in the third trimester remained

TABLE 3 Change-in-estimate of the effect of antibiotic use on wheezing outcomes in the first and third trimesters of pregnancy

\begin{tabular}{lcc} 
Confounding factors & Ever-wheezing & Recurrent wheezing \\
\hline First trimester & & -16.0 \\
$\quad$ Core confounders versus crude model & -8.7 & -15.8 \\
$\quad$ Respiratory infections versus crude model & -8.0 & -13.6 \\
$\quad$ Other infections versus crude model & -6.3 & -13.3 \\
Third trimester & & -13.5 \\
$\quad$ Core confounders versus crude model & -6.9 & -8.5 \\
$\quad$ Respiratory infections versus crude model & -9.8 & \\
$\quad$ Other infections versus crude model & -7.8 & \\
\hline
\end{tabular}

Data are presented as \%. 
TABLE 4 Associations between antibiotic treatment for genitourinary infections (recurrent urinary tract infections or Chlamydia or kidney stones) and wheezing outcomes in children aged $\leqslant 18$ months in females who had genitourinary infections during pregnancy

\begin{tabular}{|c|c|c|c|c|}
\hline & Cases & $\mathbf{R R}_{\text {crude }}$ & $\mathbf{R R}_{\text {adj1 }} \#$ & $\mathbf{R R}_{\mathrm{adj} 2}$ ๆ \\
\hline \multicolumn{5}{|l|}{ First trimester } \\
\hline Ever-wheezing $^{+}$ & 98 & & & \\
\hline No antibiotics & 18 & 1.00 (ref.) & 1.00 (ref.) & 1.00 (ref.) \\
\hline Antibiotics & 11 & $1.05(0.56-1.98)$ & $0.90(0.48-1.71)$ & $0.95(0.48-1.86)$ \\
\hline Recurrent wheezing ${ }^{\S}$ & 95 & & & \\
\hline No antibiotics & 7 & 1.00 (ref.) & 1.00 (ref.) & 1.00 (ref.) \\
\hline Antibiotics & 3 & $0.73(0.20-2.68)$ & 0.72 (0.19-2.74) & $0.93(0.18-4.72)$ \\
\hline \multicolumn{5}{|l|}{ Third trimester } \\
\hline Ever-wheezing ${ }^{f}$ & 266 & & & \\
\hline No antibiotics & 50 & 1.00 (ref.) & 1.00 (ref.) & 1.00 (ref.) \\
\hline Antibiotics & 28 & $1.06(0.72-1.55)$ & 1.06 (0.73-1.52) & $1.05(0.73-1.52)$ \\
\hline Recurrent wheezing ${ }^{\# \#}$ & 249 & & & \\
\hline No antibiotics & 14 & 1.00 (ref.) & 1.00 (ref.) & 1.00 (ref.) \\
\hline Antibiotics & 7 & $0.95(0.40-2.23)$ & $1.00(0.45-2.55)$ & $1.06(0.46-2.43)$ \\
\hline \multicolumn{5}{|c|}{ 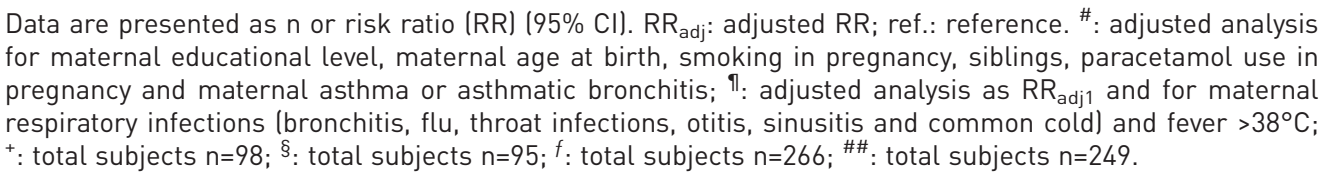 } \\
\hline
\end{tabular}

even after adjustment for a number of confounding factors. The same pattern was found when only mothers who used antibiotics for $\geqslant 3$ days or those treated with antibiotics for infections other than genitourinary ones were considered as exposed. Genitourinary infections in pregnancy were associated with an increased risk of infant wheezing, regardless of whether or not they had been treated with antibiotics.

A number of studies [5-18] assessed the association between prenatal antibiotic exposure and risk of wheezing and asthma in children. Most of them found a positive association, but the estimates were weak in some of the studies. Sтокногм and colleagues $[15,16]$ found a dose-related association between maternal antibiotic use during pregnancy and the risk of asthma in the offspring. As the associations also existed for maternal antibiotic use before and after pregnancy, they suggested that antibiotic use in pregnancy might be a proxy for mothers' general susceptibility to infection. In a large population-based registry, ÖRTQUIST et al. [17] found a positive association between prenatal antibiotic exposure and asthma in the offspring, but these findings were not confirmed in a sibling analysis, indicating a confounding role of shared familial factors. Similarly, two meta-analyses attributed a positive association between antibiotic exposure in infancy and subsequent asthma to confounding factors [30, 31].

In our analyses we accounted for the most common infections during pregnancy, and we found that respiratory and urinary infections play the strongest confounding role. Nevertheless, the role of other confounders, such as presence of siblings, cannot be neglected. Furthermore, maternal educational level, a strong determinant of socioeconomic status and access to medicines, including self-medication, has been taken into account in the analysis. However, a persistent association between antibiotic exposure during the third trimester of pregnancy and infant wheezing existed even after controlling for multiple confounders. As Caesarean section is suggested to be associated with wheezing in childhood [29], this association could be to some extent explained by the mode of delivery, as the estimate slightly decreased after exclusion of children delivered by Caesarean section. However, a positive association, although attenuated and having large confidence intervals, remained in children delivered vaginally. The role of Caesarean section in the relationship between late pregnancy antibiotic exposure and the development of wheezing in childhood still needs to be elucidated.

Six previous studies analysed exposures for each trimester of pregnancy separately and three of them $[7,11,18]$ found the strongest associations for exposure in late pregnancy, especially during the third trimester. In the other three studies [6, 15-17] associations did not depend on the period of exposure. The composition of the maternal commensal flora, which is influenced by antibiotic use in late pregnancy [32], may modify the maturation of the newborn immune system by altering normal microbial colonisation of the infant gut or airways [19]. These alterations of the immune system might lead to an atopic phenotype and an increased susceptibility to infections/wheezing. 
In addition, we found an increased risk of infant wheezing after maternal genitourinary infections in pregnancy, regardless of antibiotic use, suggesting that these infections per se might be associated with wheezing in offspring. Consistently, in a previous cross-sectional study [8], antibiotics administered for maternal urinary infections were associated with transient early childhood wheezing, and an association between maternal urinary infections during pregnancy and childhood asthma has been found in two cohort studies $[13,33]$. Abnormal colonisation of the genitourinary tract and ascending infections are possibly a proxy of chorioamnionitis [34], which in turn could play a role in modulating lung development [22].

Our study had some limitations. The NINFEA questionnaires do not include information on type of antibiotics administered, and analysing them all together yields an "average" effect of antibiotics, preventing us from estimating possibly diverse effects of different antibiotic classes, and of the mechanisms involved. Restriction of the exposed population to mothers who took antibiotics for infections other than genitourinary ones, assuming that most of them suffered from respiratory infections and hence used broad-spectrum antibiotics, did not change the results.

Our study is based on self-reported information, which may introduce misclassification. However, misclassification of the exposure was most probably driven by underreporting, rather than overreporting, which translates into high specificity and a reduced impact of nondifferential misclassification for an exposure with 6-7\% prevalence [35]. Moreover, analyses restricted to mothers who used antibiotics for $\geqslant 3$ days and to those treated for genitourinary infections, designed to have a higher specificity and less potential for bias from nondifferential misclassification, did not alter the results.

We also relied on self-reported information when defining the outcome. Wheezing reported by mothers, although widely accepted in large epidemiological studies, might overestimate the presence of the condition, in particular ever-wheezing, as parents might label a single episode of noisy breathing as wheezing [36]. Recurrent wheezing is less affected by this source of bias and is more likely to identify a pathological condition [36].

As we studied children aged $\leqslant 18$ months we were unable to distinguish different wheezing phenotypes. Follow-up at older ages is therefore needed in order to clarify the role of prenatal antibiotics, if any, in the development of asthma and wheezing persisting into school age.

Another limitation is that the associational estimate between the third trimester exposure and recurrent wheezing might be affected by recall bias, as this exposure is assessed at the 6-month questionnaire together with wheezing in the first 6 months of life, which is a necessary component of the definition of recurrent wheezing. This could be relevant as we found an effect for the third trimester exposure only.

As in many other cohort studies, members of the NINFEA cohort mainly originate from a selected population with high education and socioeconomic status. However, it has been shown extensively that this baseline selection does not imply biased associational estimates in cohort studies [37, 38]. Furthermore, in the NINFEA cohort, the distribution of the exposure and outcomes did not differ between different levels of education.

In conclusion, the associations between prenatal antibiotic exposure and early childhood wheezing are largely explained by confounding factors, in particular maternal infections during pregnancy. Although multiple adjustments and the exclusion of children delivered by Caesarean section attenuated the magnitude of the association, the risk of recurrent wheezing after maternal antibiotic use in the third trimester remained increased. Our data also suggest a positive association between genitourinary infections during pregnancy and infant wheezing, independently of antibiotic use. These results need replication as our analysis was not originally designed to address this specific association.

\section{References}

1 Ducharme FM, Tse SM, Chauhan B. Diagnosis, management, and prognosis of preschool wheeze. Lancet 2014; 383: 1593-1604.

2 Duijts L. Fetal and infant origins of asthma. Eur J Epidemiol 2012; 27: 5-14.

3 Silvestri M, Franchi S, Pistorio A, et al. Smoke exposure, wheezing, and asthma development: a systematic review and meta-analysis in unselected birth cohorts. Pediatr Pulmonol 2015; 50: 353-362.

4 Fuchs O, von Mutius E. Genetic and environmental factors in bronchial asthma and wheezing disorders. In: Eber E, Midulla F, eds. ERS Handbook of Paediatric Respiratory Medicine. 1st Edn. Sheffield, European Respiratory Society, 2013; pp. 298-304.

5 Benn CS, Thorsen P, Jensen JS, et al. Maternal vaginal microflora during pregnancy and the risk of asthma hospitalization and use of antiasthma medication in early childhood. J Allergy Clin Immunol 2002; 110: 72-77.

6 McKeever TM, Lewis SA, Smith C, et al. The importance of prenatal exposures on the development of allergic disease: a birth cohort study using the West Midlands General Practice Database. Am J Respir Crit Care Med 2002; 166: 827-832.

7 Jedrychowski W, Galaś A, Whyatt R, et al. The prenatal use of antibiotics and the development of allergic disease in one year old infants. A preliminary study. Int J Occup Med Environ Health 2006; 19: 70-76. 
8 Rusconi F, Galassi C, Forastiere F, et al. Maternal complications and procedures in pregnancy and at birth and wheezing phenotypes in children. Am J Respir Crit Care Med 2007; 175: 16-21.

9 Martel MJ, Rey E, Beauchesne MF, et al. Control and severity of asthma during pregnancy are associated with asthma incidence in offspring: two-stage case-control study. Eur Respir J 2009; 34: 579-587.

10 Dom S, Droste JH, Sariachvili MA, et al. Pre- and post-natal exposure to antibiotics and the development of eczema, recurrent wheezing and atopic sensitization in children up to the age of 4 years. Clin Exp Allergy 2010; 40: $1378-1387$

11 Stensballe LG, Simonsen J, Jensen SM, et al. Use of antibiotics during pregnancy increases the risk of asthma in early childhood. J Pediatr 2013; 162: 832-838.

12 Källén B, Finnström O, Nygren KG, et al. Maternal drug use during pregnancy and asthma risk among children. Pediatr Allergy Immunol 2013; 24: 28-32.

13 Collier $\mathrm{CH}$, Risnes $\mathrm{K}$, Norwitz ER, et al. Maternal infection in pregnancy and risk of asthma in offspring. Matern Child Health J 2013; 17: 1940-1950.

14 Metsälä J, Lundqvist A, Virta LJ, et al. Prenatal and post-natal exposure to antibiotics and risk of asthma in childhood. Clin Exp Allergy 2015; 45: 137-145.

15 Stokholm J, Sevelsted A, Bønnelykke K, et al. Maternal propensity for infections and risk of childhood asthma: a registry-based cohort study. Lancet Respir Med 2014; 2: 631-637.

16 Bisgaard H, Stokholm J, Sevelsted A, et al. Maternal antibiotic use and risk of asthma in offspring - Authors' reply. Lancet Respir Med 2014; 2: e17.

17 Örtqvist AK, Lundholm C, Kieler H, et al. Antibiotics in fetal and early life and subsequent childhood asthma: nationwide population based study with sibling analysis. BMJ 2014; 349: g6979.

18 Lapin B, Piorkowski J, Ownby D, et al. Relationship between prenatal antibiotic use and asthma in at-risk children. Ann Allergy Asthma Immunol 2015; 114: 203-207.

19 Azad MB, Kozyrskyj AL. Perinatal programming of asthma: the role of gut microbiota. Clin Dev Immunol 2012; 2012: 932072.

20 Bisgaard H, Hermansen MN, Buchvald F, et al. Childhood asthma after bacterial colonization of the airway in neonates. N Engl J Med 2007; 357: 1487-1495.

21 Murk W, Risnes KR, Bracken MB. Prenatal or early-life exposure to antibiotics and risk of childhood asthma: a systematic review. Pediatrics 2011; 127: 1125-1138.

22 Kumar R, Yu Y, Story RE, et al. Prematurity, chorioamnionitis, and the development of recurrent wheezing a prospective birth cohort study. J Allergy Clin Immunol 2008; 121: 878-884.

23 Richiardi L, Baussano I, Vizzini L, et al. Feasibility of recruiting a birth cohort through the Internet: the experience of the NINFEA cohort. Eur J Epidemiol 2007; 22: 831-837.

24 Bajardi P, Paolotti D, Vespignani A, et al. Association between recruitment methods and attrition in Internet-based studies. PLoS One 2014; 9: e114925.

25 Cummings P. Methods for estimating adjusted risk ratios. Stata J 2009; 9: 175-196.

26 Greenland S. Introduction to regression modeling. In: Rothman KJ, Greenland S, Lash TL, eds. Modern Epidemiology. 3rd Edn. Philadelphia, Lippincott Williams \& Wilkins, 2008; pp. 419-421.

27 O'brien RM. A caution regarding rules of thumb for variance inflation factors. Qual Quant 2007; 41: 673-690.

28 National Institute for Health and Care Excellence. Caesarean section (update). (Clinical guideline 132). 2011. http://guidance.nice.org.uk/CG132. Date last accessed: June 10, 2014.

29 Thavagnanam S, Fleming J, Bromley A, et al. A meta-analysis of the association between Caesarean section and childhood asthma. Clin Exp Allergy 2008; 38: 629-633.

30 Marra F, Lynd L, Coombes M, et al. Does antibiotic exposure during infancy lead to development of asthma? A systematic review and metaanalysis. Chest 2009; 136: Suppl. 5, e30.

31 Penders J, Kummeling I, Thijs C. Infant antibiotic use and wheeze and asthma risk: a systematic review and meta-analysis. Eur Respir J 2011; 38: 295-302.

32 Stokholm J, Schjørring S, Eskildsen CE, et al. Antibiotic use during pregnancy alters the commensal vaginal microbiota. Clin Microbiol Infect 2014; 20: 629-635.

33 Algert CS, Bowen JR, Lain SL, et al. Pregnancy exposures and risk of childhood asthma admission in a population birth cohort. Pediatr Allergy Immunol 2011; 22: 836-842.

34 Tita AT, Andrews WW. Diagnosis and management of clinical chorioamnionitis. Clin Perinatol 2010; 37: 339-354.

35 Rothman KJ, Greenland S, Lash TL. Validity in epidemiological studies. In: Rothman KJ, Greenland S, Lash TL, eds. Modern Epidemiology. 3rd Edn. Philadelphia, Lippincott Williams \& Wilkins, 2008; pp. 139-142.

36 Brand PL, Baraldi E, Bisgaard H, et al. Definition, assessment and treatment of wheezing disorders in preschool children: an evidence-based approach. Eur Respir J 2008; 32: 1096-1110.

37 Rothman KJ, Gallacher JE, Hatch EE. Why representativeness should be avoided. Int J Epidemiol 2013; 42: 1012-1014.

38 Pizzi C, De Stavola BL, Pearce N, et al. Selection bias and patterns of confounding in cohort studies: the case of the NINFEA web-based birth cohort. J Epidemiol Community Health 2012; 66: 976-981. 